\title{
Texture-controlled hybrid materials fabricated using nanosecond technology
}

\author{
Hong-Baek CHO, ${ }^{* * *}$ Tadachika NAKAYAMA, ${ }^{, \dagger}$ Minh Triet Tan HUYNH, ${ }^{*}$ \\ Son Thanh NGUYEN, ${ }^{*}$ Weihua JIANG, ${ }^{*}$ Tsuneo SUZUKI, ${ }^{*}$ Hisayuki SUEMATSU, ${ }^{*}$ \\ Koichi NIIHARA, ${ }^{*}$ Jung Ho SHIN*** and Yong-Ho CHOA $^{* *, *}$ \\ ${ }^{*}$ Extreme Energy-Density Research Institute, Nagaoka University of Technology, \\ 1603-1 Kamitomioka, Nagaoka, Niigata 940-2188, Japan \\ ** Department of Materials Science \& Chemical Engineering, Hanyang University, Ansan, Gyeonggi 426-791, Korea \\ *** Division of Materials Science and Engineering, Pusan National University, Busan 609-735, Korea
}

\begin{abstract}
The controlled assembly of micro- and nano-ceramic fillers in polymer nanocomposites provides robust properties such as wetting, adhesion, thermal conductivity, electrical insulation and optical activity, and enable the extended application of these hybrid materials as thermal interfacing materials in microelectronics and for energy conversion. However, the required properties can only be obtained either by homogeneous mixing or by anisotropic orientation of a large amount $(>50 \mathrm{vol} . \%)$ of expensive fillers, which is economically inefficient. Here we propose a strategy for tuning the orientation and assembly of ceramic boron nitride nanofillers in a polymer nanocomposite using a small amount $(<5 \mathrm{vol} . \%)$ of filler to enhance thermal conduction. The texture of the BN fillers is tuned by application of a nanosecond pulse electric field and a superconductor magnetic field $(10 \mathrm{~T})$; the three-dimensional structure of the products was analyzed using 3-D X-ray CT scanning. The enhanced anisotropic orientation and thermal properties of the products were assessed as a function of the structural variation of the boron nitride fillers in the polymer.
\end{abstract}

(02016 The Ceramic Society of Japan. All rights reserved.

Key-words : Boron nitride, Polysiloxane, Nanosecond pulse electric field, Magnetic field

[Received September 25, 2015; Accepted December 30, 2015]

\section{Introduction}

Polymer nanocomposites with high electrical insulation and thermal conductivity properties are promising materials for micro- and nano-electronics applications such as sensors, actuators, and solar cells. ${ }^{1)-3)}$ However, the ongoing miniaturization and integration of power-intensive devices results in thermal management problems affecting the performance, reliability and stabilization of devices. ${ }^{4)}$ For example, the temperature rise in photovoltaic solar cells reduces the produced power by $\sim 20 \%$ due to heat build-up and corresponds to an efficiency loss of $0.5 \%$ per $1{ }^{\circ} \mathrm{C}$ increase in the temperature of the cell. ${ }^{5)}$

Materials ideal for effective thermal management should have high thermal conductivity and high electrical insulation. Thermal interface materials (TIM) are positioned between the heat generating source and the heat sink and minimize thermal buildup by effectively transferring the generated heat to the heat sink. TIMs with these properties have been generated either by homogeneous mixing or by anisotropic orientation of large amounts ( $>50 \mathrm{vol} \%$ ) of expensive fillers, ${ }^{6}$ ) and their extended application is limited due to the decreased workability of the composite films and the high cost of the fillers.

Aligned one-dimensional (1-D) or two-dimensional (2-D) nanofillers in a polymer matrix exhibit conductivity percolation using a smaller amount of fillers compared to composites with

Corresponding author: T. Nakayama; E-mail: nky15@vos. nagaokaut.ac.jp

* Corresponding author: Y.-H. Choa; E-mail: choa15@hanyang. ac.kr randomly distributed fillers. ${ }^{7), 8}$ Furthermore, the physical, electrical and thermal properties of pristine polymers can be modified by tuning the orientation and assembly of inorganic inclusions. Vertical double percolation (VDP), ${ }^{9)}$ a honeycomb-like structure, ${ }^{10)}$ and self-assembly ${ }^{11)}$ techniques have been applied to the controlled assembly of 1-D and 2-D nanofillers. VDP and selfassembly alter the interactions between fillers by controlling their electrical or chemical properties, whereas the honeycomb-like technique uses polymer balls as a matrix to facilitate the physical separation of domains between the polymer and inorganic boron nitride $(\mathrm{BN})$ fillers prior to thermal treatment. ${ }^{10)}$ Electric fields, ${ }^{12)}$ shear forces ${ }^{13)}$ and magnetic fields ${ }^{14)}$ have been applied as external torque sources for the anisotropic orientation of nanofillers.

Electrophoresis, polarization, Coulombic attraction, surface modification and aspect ratio have been used to determine the anisotropic orientation and assembly of 1-D/2-D nanofillers in polymer matrices in an electric field. ${ }^{15)}$ The electric field should be as high as possible in order to control the alignment and assembly of the insulating ceramic fillers in an amorphous polymer matrix. However, the intensity of the electric field that can be applied to the composite system is limited by electrical breakdown and burnout of the material caused by application of an electric field intensity higher than the breakdown voltage. ${ }^{15)}$ We recently reported that a nanosecond pulse electric field does not cause electrical breakdown, yet orients insulating BN nanosheets: ${ }^{15), 16)}$ electrical breakdown caused by application of $1 \mathrm{kV} \mathrm{DC}$ did not occur when up to a $40 \mathrm{kV}$ nanosecond pulse electric field was applied. The anisotropic orientation of the $\mathrm{BN}$ nanosheets was demonstrated by activating the diamagnetic 
susceptibility of the $c$-axis of the BN using a high (10 T) magnetic field. ${ }^{17)}$ The combination of nanosecond pulse electric field application coupled with diamagnetic susceptibility measurements showed that the insulating ceramic BN fillers can be oriented without the need for surface modification. However, structural variations of the $\mathrm{BN}$ fillers in the polymer, and the effect of these variations on the thermal conduction properties during application of both nanosecond pulse electric fields and magnetic fields, need to be understood before these materials can be used for practical applications.

We here investigated controlling the texture of insulating BN fillers in a polymer using two torque sources: a nano pulse electric field and a magnetic field. The effects of the two fields on 3-D structural variations of the fillers were determined using Xray computed tomography and thermal diffusivity was evaluated. This strategy for tuning the insulating and diamagnetic susceptibility of 1-D or 2-D in a polymer matrix may help enhance the thermal and optical properties of hybrid materials using small amounts of nanofillers.

\section{Experimental}

\subsection{Materials}

Nano-dimensional hexagonal $\mathrm{BN}$ nanosheets (BNNSs) were purchased (DENKA Co., Ltd.) and used as received. Two polysiloxane pre-polymers with different viscosities were used: silicone YE5822(A), 1.2 Pa S, and silicone YE5822(B), 0.2 Pa $\mathrm{S}$ (Momentive Co., Ltd.). An indium-tin oxide (ITO) coated glass slide with $8-12 \Omega /$ sq surface resistivity (Sigma-Aldrich Co.) was used as an electrode for application of the electric field. The silicone release agents HD-1101 TH and HD-TH (Harves Co. Ltd.) were used on the surface of the ITO-coated electrode to aid detachment of the composite film.

\subsection{Localization control of $\mathrm{BN}$ nanosheets in polymer-based nanocomposite films}

Polysiloxane/BNNSs hybrid nanocomposites were fabricated by application of a nanosecond pulse electric field as described previously. ${ }^{18)}$ Briefly, a pre-polymer mixture of silicone YE5820(A) and YE5822(B) was prepared by mixing $3.0 \mathrm{~g}$ of silicone YE5820(A), $0.3 \mathrm{~g}$ of silicone YE5822(B) and $0.372 \mathrm{~g}$ of hexagonal $\mathrm{BN}(h-\mathrm{BN})(10 \mathrm{vol} . \%)$ in a high-speed mixer at $1500 \mathrm{rpm}$ for $5 \mathrm{~min}$ to produce a homogeneous dispersion. This dispersion was cast onto a glass spacer $\left(15 \times 15 \mathrm{~mm}^{2} \times 250\right.$ $\mu \mathrm{m})$ precoated first with the silicone release agent HD-1101 TH, then with HD-TH (soaking for $4 \mathrm{~s}$ in the silicone, then drying for $2 \mathrm{~min}$ ), which was then located between two ITO coated glass electrodes. The cast polymer was kept for $16 \mathrm{~h}$ under a nanosecond pulse electric field applied in one direction (single direction). Alternatively, the polarity of the nanopulse electric field was switched once after $8 \mathrm{~h}$ to observe changes in the movement of the nanofillers in the polymer; this approach was termed 'nano pulse-polarity change'. The fabricated composite films are detached from the electrodes and underwent drying for $30 \mathrm{~min}$ at $80^{\circ} \mathrm{C}$ prior to recovery. The orientation of the BNNSs under application of a high magnetic field was conducted as described elsewhere: ${ }^{17}$ ) the pre-polymer suspension was subjected to a $10 \mathrm{~T}$ magnetic field (a superconducting magnet) for $24 \mathrm{~h}$ to enhance either the perpendicular or parallel alignment of the BN nanosheets in the composite film. The polysiloxane/BN nanosheet composites were dried for $0.5 \mathrm{~h}$ at $80^{\circ} \mathrm{C}$ to complete curing. Figure 1 illustrates the experimental setup used to apply the electric fields. The amount of BN was varied from $0-15$ vol.\% whereas the frequency of the nanopulse-wide field was fixed at
$10 \mathrm{~Hz}$. The nanopulses were controlled at $50 \mathrm{~ns} / \mathrm{dV}$ in a $40 \mathrm{kV}$ electric field.

\subsection{Analysis and characterization}

The anisotropic alignment of BNNSs in the composite films was analyzed using an X-ray diffractometer (XRD, RINT 2500, Rigaku, Co.). The degree of anisotropic alignment of the BNNSs perpendicular to the film plane in the prepared samples was estimated by comparing the percent ratio calculated by $a$-axis/ $(a$-axis $+c$-axis $) \times 100$. The film thickness and surface morphology of the composites were examined using a scanning electron microscope (SEM) (JSM-6700F.JEOL, Ltd.). The 3-D distribution of BNNS in the matrix was observed with a high resolution X-ray micro CT scanner (SkyScan 1172, Bruker micro $\mathrm{CT}$, detail detectability; $0.5 \mu \mathrm{m}$ at highest resolution) using the following settings: $60 \mathrm{kV} \mathrm{X-ray} \mathrm{energy,} 1350 \mathrm{~ms}$ exposure time, $0.25 \mathrm{deg}$. rotation step. The 3 -D X-ray tomographic images were obtained by reconstructing all the observed X-ray transmission section images.

\section{Results and discussion}

\subsection{Structural variation in BNNS assembly due to different external torque sources}

Figure 2 shows magnified images of hexagonal BNNSs and a fabricated polysiloxane/BNNS composite. The graphite-like layered structure of $\mathrm{BN}$ has a smooth, round shape and the longitudinal length ranges from 5 to $20 \mu \mathrm{m}$ [Fig. 2(a)]. The thickness of the $\mathrm{BN}$ ranges from 5 to $15 \mathrm{~nm}$, depending on the number of the h-BN layers [Fig. 2(b)]. The inset shows the fabricated polymer/BNNS nanocomposite film $\left(15 \times 15 \mathrm{~mm}^{2} \times 250 \mu \mathrm{m}\right)$. The flexibility of the composite allows its extended application on various protrusions micro- and nano-electronic surfaces.

The X-ray diffraction patterns of polysiloxane/BNNS composite films as a function of fabrication conditions are shown in Fig. 3. Random distribution of the BNNSs provides a high BN intensity of (002) at $2 \theta=26.75$ in the polymer matrix; this intensity decreases as the $\mathrm{BN}$ plane is oriented perpendicular to the composite film plane, providing an increase in the (100) intensity at $2 \theta=41.58$. The intensity ratio (I.R.) is calculated by comparing the intensities of the $c$-axis, (002) plane and $a$-axis, (100) plane, and describes the degree of BNNS plane orientation vertical to the film planes under conditions of high BNNS anisotropy. The composite with random BNNS distribution shows an I.R. of $4.43 \%$, which decreases to $3.19 \%$ when the BNNSs are oriented parallel to the film plane. When the BNNSs are oriented perpendicular to the film plane under a magnetic field, the I.R. becomes maximum $(49.21 \%)$ compared to other external orientation sources, electric fields. The nanosecond pulse electric field in one direction increased I.R. to $30.56 \%$ (described as Nano pul.-single direction) and to $44.41 \%$ upon polarity change of the nanosecond pulse electric field (described as Nano pul.-polarity change). Thus, by changing the direction of the nanosecond pulse for the $16 \mathrm{~h}$ electric field application, the I.R. was enhanced by $14 \%$ compared to single-direction application. The low I.R. value obtained for the parallel orientation and the high I.R. value obtained for the perpendicular orientation of BNNSs indicates that application of a high magnetic field (10 T) is more effective for controlling the orientation of BNNSs in polysiloxane matrix under a given condition compared to the application of a nanosecond pulse electric field. The orientation of $h$-BN under a high magnetic field relates to the diamagnetic susceptibility of $h$-BN along the $c$-axis, which is $-0.48 \times 10^{-6} \mathrm{Am}^{2} / \mathrm{kg}$. ${ }^{19)}$ The nitrogen atoms engaged in double bonds in $h$-BN carry a positive charge due to $\pi$ - 
a)

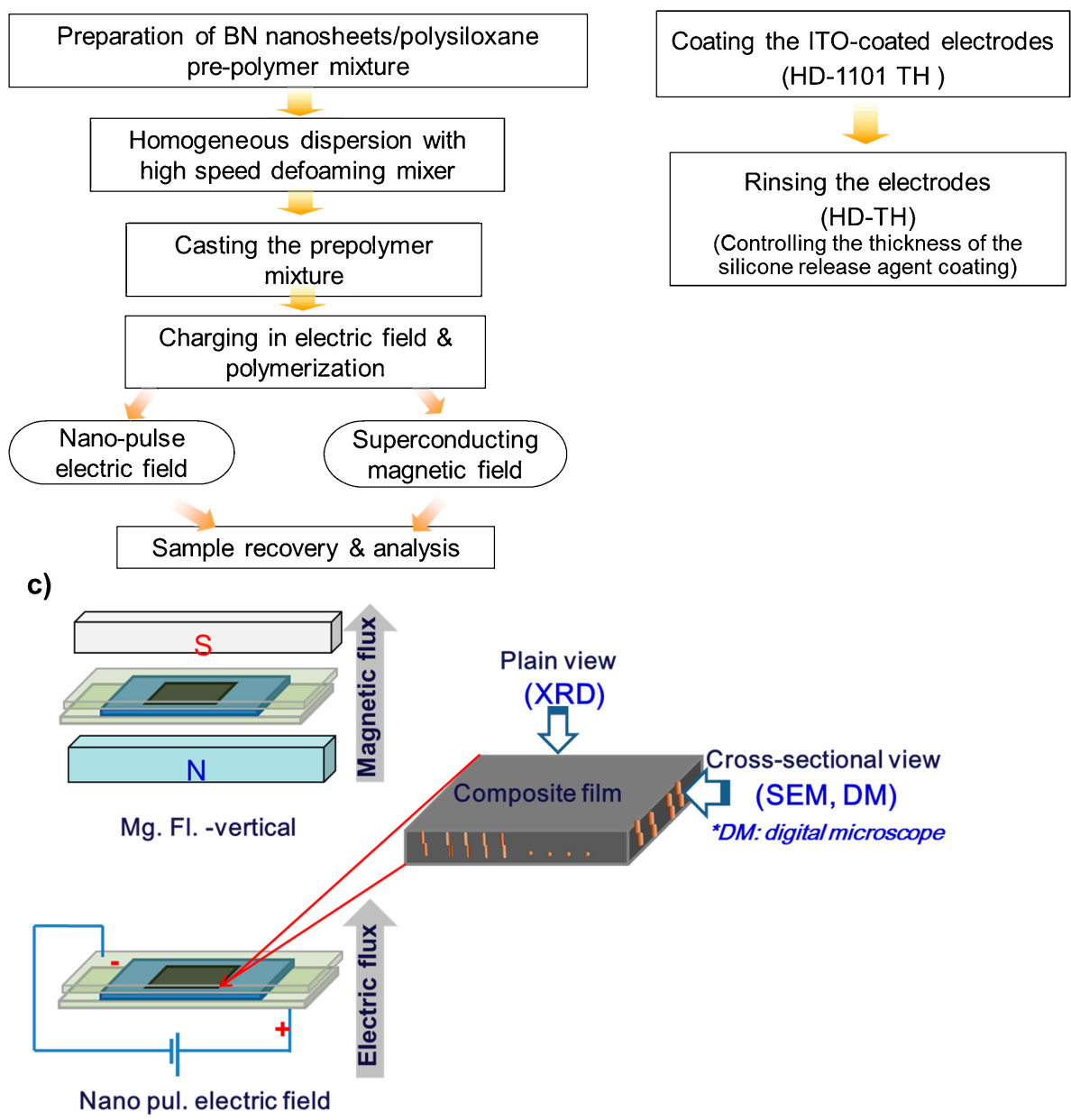

b) 
domains in the LBBNs ( $\mathrm{x}-\mathrm{z}$ plane tomography) increased if the nanosecond pulse electric field changed polarity. Comparison of the 3-D images in Figs. 5(c) and 5(d) confirms the development LBBNS from linear branches of BNNSs.

\subsection{Thermal properties}

Figure 6 shows the thermal diffusivity of composite films as a function of the fabrication conditions. The influence of the degree of orientation and the structural variation of BNNSs on the
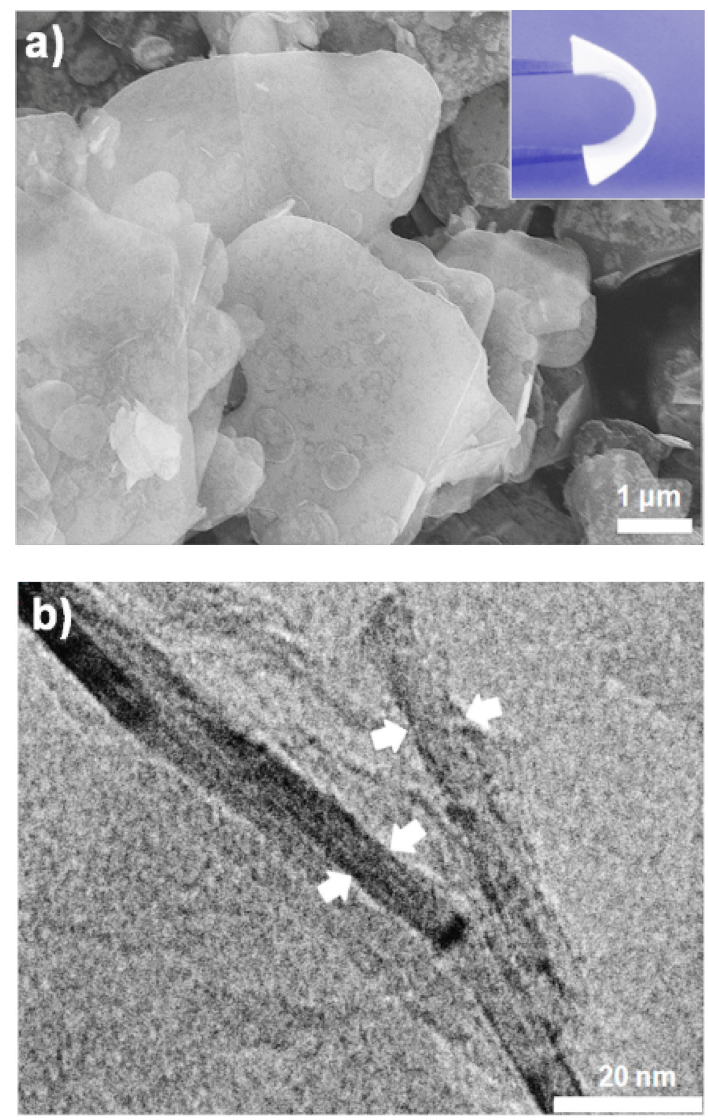

Fig. 2. Micrographs of BNNs by a) SEM (inset: fabricated nanocomposite film with flexibility with incorporation of BNNSs $[15 \times$ $15 \mathrm{~mm}^{2} \times 250 \mu \mathrm{m}(\mathrm{BN} 15$ vol. \%)] and by b) TEM. thermal properties of the polymer/BNNS composites were compared. The thermal diffusivity of pristine polysiloxane is $1.66 \mathrm{E}-07\left(\mathrm{~m}^{2} \mathrm{~s}^{-1}\right)$; this increased to $1.97 \mathrm{E}-07\left(\mathrm{~m}^{2} \mathrm{~s}^{-1}\right)$ by the addition of $5 \mathrm{vol} \% \mathrm{BN}$, but decreased to $1.82 \mathrm{E}-07\left(\mathrm{~m}^{2} \mathrm{~s}^{-1}\right)$ under application of a nano pulse-single direction electric field and to $1.75 \mathrm{E}-07\left(\mathrm{~m}^{2} \mathrm{~s}^{-1}\right)$ under application of a magnetic field, causing the BNNSs to orient parallel to the film plane. The thermal diffusivity increased to $2.33 \mathrm{E}-07\left(\mathrm{~m}^{2} \mathrm{~s}^{-1}\right)$ following perpendicular orientation of the BN planes and to $5.32 \mathrm{E}-07\left(\mathrm{~m}^{2} \mathrm{~s}^{-1}\right)$ by application of a nano pulse-polarity change electric current. This later value $\left[5.32 \mathrm{E}-07\left(\mathrm{~m}^{2} \mathrm{~s}^{-1}\right)\right]$ is $320 \%$ higher than that of pristine polysiloxane and $252 \%$ higher that then of the corresponding composite with randomly distributed BNNs. The highest thermal

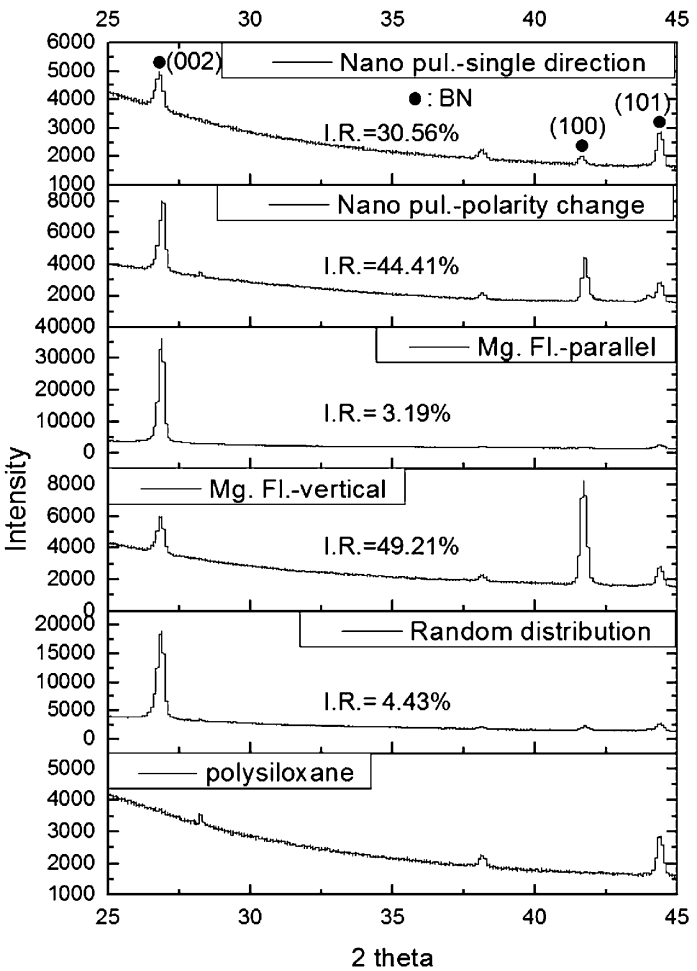

Fig. 3. X-ray diffraction patterns of polysiloxane/BNNS composite films as a function of fabrication conditions. \{Intensity ratio $(\%)=$ $[a$ axis $/(a$ axis $+c$ axis $)] \times 100(\%)\}$
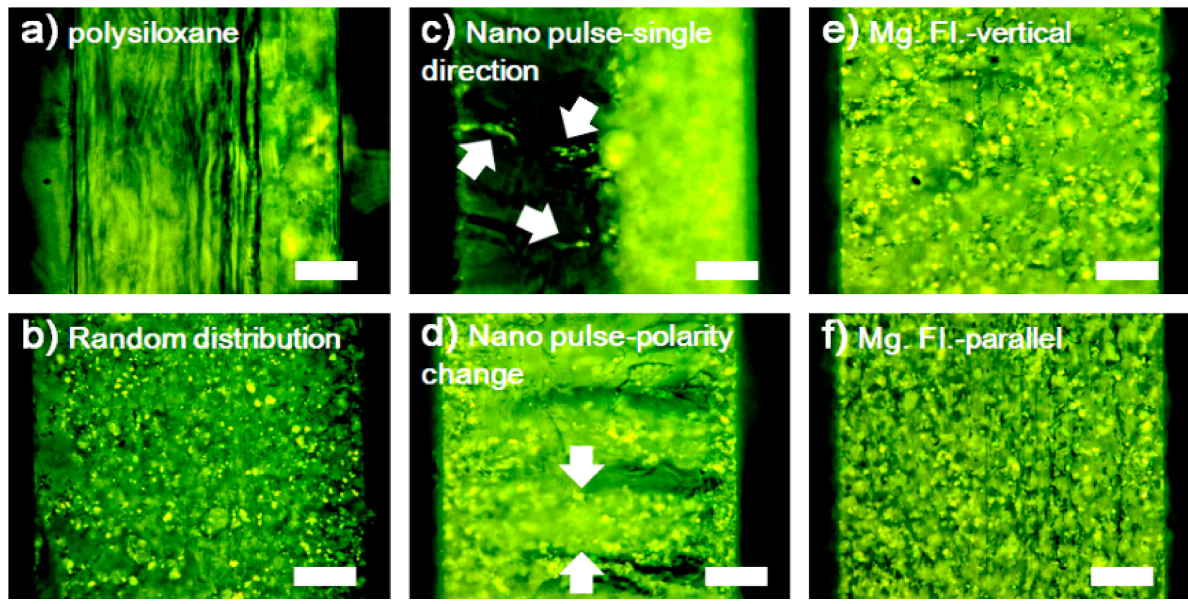

Fig. 4. Cross-sectional 2-D analysis of polysiloxane/BNNS composite films using a digital microscope (BN, 5 vol.\%; scale bar, $50 \mu \mathrm{m}$ ): a) polysiloxane, b) random distribution, c) nano pulse-single direction, d) nano pulse-polarity change, e) magnetic field-vertical and f) magnetic field-parallel. 
a)
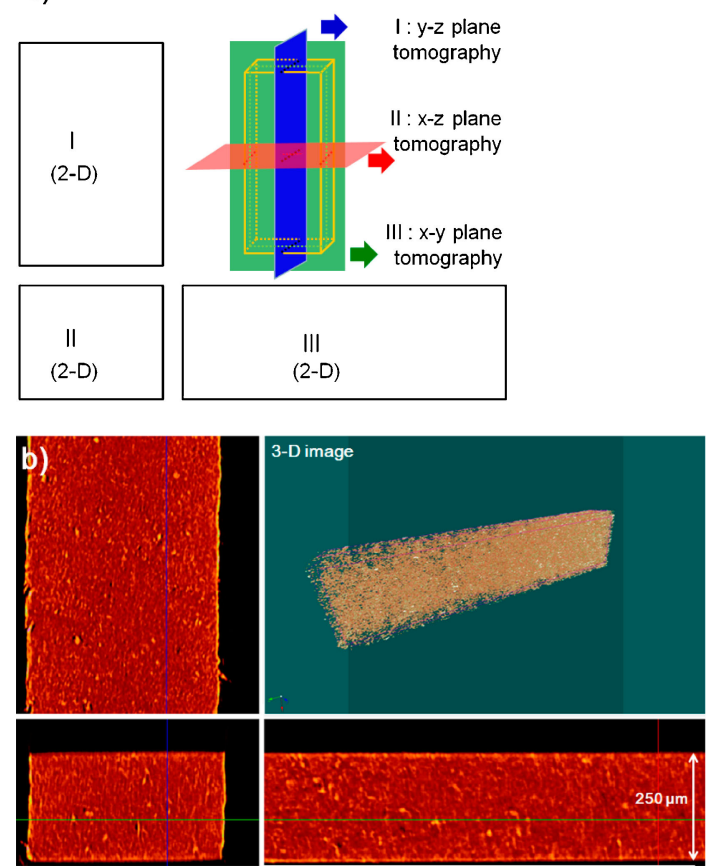
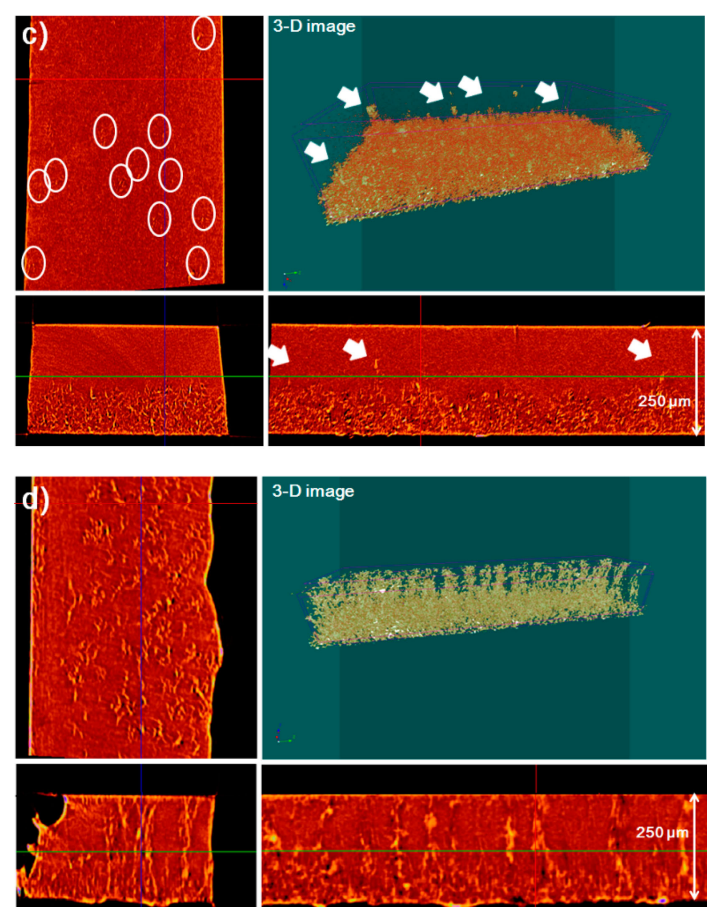

Fig. 5. 2-D and 3-D analysis of polysiloxane/BNNS composite films by 3-D X-ray CT scanning (BN, 5 vol.\%): a) schematic of 2-D tomographic images (All three images; I, II and III planes, are cross-sectional views of each sample using 3-D X-ray CT scanning from three different planes), b) random distribution, c) nano pulse- single direction and d) nano pulse-polarity change.

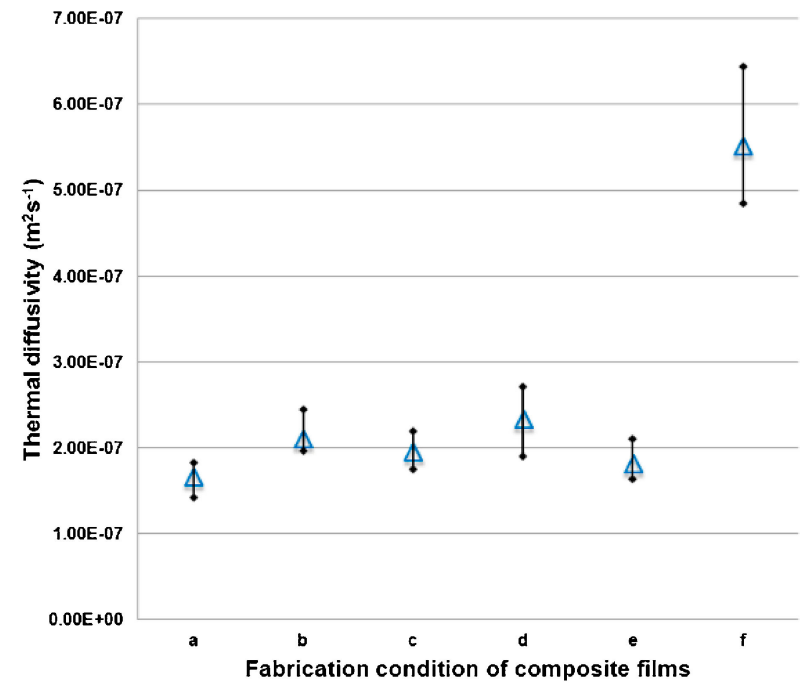

Fig. 6. Thermal diffusivity of polysiloxane/BNNS composite films (BN, 5vol.\%; measurement, out-of-plane direction): a) polysiloxane, b) random distribution, c) nano pulse-single direction, d) magnetic fieldvertical, e) magnetic field-parallel and f) nano pulse-polarity change.

diffusivity was obtained for composites prepared using a nano pulse-polarity change current.

\subsection{Techniques for texture control of hybrid nano- composites in relation to enhanced thermal properties}

The anisotropic orientation of the BNN planes either parallel or perpendicular to the film plane was confirmed using a $10 \mathrm{~T}$ magnetic field. The magnetic field was more effective at aligning the BNN planes than the nanosecond electric pulse technique. The application of a nano single-direction pulse facilitated localization of the BNNSs to the positive electrode and formation of linear branched BNNSs which developed into LBBNs connecting the film surfaces. Importantly, electrophoresis and controlled orientation of BNNSs was achieved without the need to modify the BN surface with magnetic metal nanoparticles ${ }^{21)}$ or by chemical functionalization. ${ }^{22}$ It is noteworthy that the breakdown of polysiloxane-based composite $250 \mu \mathrm{m}$ thick occurred using an electric field of less than DC $3.5 \mathrm{kV}$, which was verified to be overcome by applying electric field with nanopulse intervals, ${ }^{18)}$ as is the demonstration of the morphological changes of $h$-BN nanofillers into the linear branched structure and the thicker branched structure using further modified techniques. The generation of the linear bridged structure of $\mathrm{BN}$ by polarity change may be explained by coordinated effects of dipolarization, Columbic attraction, electrophoresis, and surface charge influenced by static electricity, as mentioned in related research on the incorporation of micro-diamond fillers into polymer matrices. ${ }^{23)}$ Despite the very low dielectric constant of insulating $h$-BN $(\approx 2.3)^{24)}$ and the requisite very high electric voltage required compared to conducting graphite nanosheets (dielectric constant $\approx 13.5$ ), ${ }^{25)}$ a $40 \mathrm{kV}$ electric field pulsed at $50 \mathrm{~ns}$ intervals is sufficient to drive filler-to-filler contact, resulting in the fabrication of a thicker and denser LBBN structure by nano pulse-polarity change.

Thermal conduction in the out-of-plane direction of the composite due to vertical orientation of the BNNSs was higher than when the BNNSs were randomly distributed, indicating that higher, more efficient thermal conduction might be realized by further facilitating filler-to-filler connections, as demonstrated by application of nano pulse-polarity change.

\section{Conclusion}

The texture of insulating BN nanofillers in a polymer-based hybrid material was controlled using either a nano-pulse electric field or superconducting magnetic fields. The analytical results 
of XRD and digital microscope exhibited that application of a $10 \mathrm{~T}$ magnetic field tuned the anisotropic alignment of BNNSs to either perpendicular or parallel to the composite film surface, and the additional coordination of 2-D and 3-D structural analysis by 3-D X-ray CT scanning revealed that application of a nano-pulse electric field with polarity change facilitated the formation of a linear bridged structure of BNNSs (LBBNs) in the polymer without causing electrical breakdown. The presence of LBBNs in the composites increased the thermal diffusivity to 5.32E-07 $\left(\mathrm{m}^{2} \mathrm{~s}^{-1}\right)$, which is 2.5 times higher than that of the composite exhibiting the highest perpendicular anisotropic orientation to the film under application of a magnetic field-inducement (2.33E-07 $\left.\mathrm{m}^{2} \mathrm{~s}^{-1}\right)$. This demonstrated that inducing contacts between fillers with a linear structure is a more straightforward approach for enhancing thermal dissipation to out-of-plane composite films than enhancing the perpendicular orientation of fillers with no inter-particle connections. Thus, alignment technology using polarity change of a nanosecond pulse electric field holds potential for tuning the orientation and assembly of insulating or diamagnetic nanofillers in a polymer matrix, and thus may be promising for application in the electronics, optoelectronics and semiconductor industries for the generation of photonic devices and systems.

Acknowledgement This research was supported by Basic Science Research Program through the National Research Foundation of Korea (No. 2015R1A5A1037548) and the Fundamental R\&D Program for Core Technology of Materials (10050890, Chalcogenide nanostructure-based room-temperature $\left(25^{\circ} \mathrm{C}\right) \mathrm{H}_{2} \& \mathrm{H}_{2} \mathrm{~S}$ gas sensors with low power consumption) and the Human Resources Development program (No. 20154030200680) of the Korea Institute of Energy Technology Evaluation and Planning.

\section{References}

1) K. Asokan, J. Y. Park, S.-W. Choi and S. S. Kim, Nanoscale Res. Lett., 5, 747-752 (2010).

2) F. C. Fim, J. M. Guterres, N. R. S. Basso and G. B. Galland, J. Polym. Sci. Part A: Polym. Chem., 48, 692-698 (2010).

3) R. Haggenmueller, C. Guthy, J. R. Lukes, J. E. Fisher and K. I. Winey, Macromolecules, 40, 2417-2421 (2007).

4) Q. Liang, X. Yao, W. Wang, Y. Liu and C. P. Wong, ACS Nano, 5, 2392-2401 (2011).

5) K. M. F. Shahil and A. A. Balandin, Solid State Commun., 152, 1331-1340 (2012).

6) K. Miyata, T. Yamagata and T. Adschiri, in 10th international symposium on advanced organic photonics \& 1st international symposium on super-hybrid materials 74 (Tokyo \& Sendai, Japan; 2010).

7) X. S. Du, M. Xiao, Y. Z. Meng and A. S. Hay, Polymer, 45, 6713-6718 (2004).

8) D. W. Liu, X. S. Du and Y. Z. Meng, Polym. Polym. Compos., 13, 815-821 (2005).

9) D. Yorifuji and S. Ando, Macromol. Chem. Phys., 211, 21182124 (2010).

10) Y. Agari, A. Ueda and S. Nagai, J. Appl. Polym. Sci., 49, 16251634 (1993).

11) T. Prasse, J.-Y. Cavaillé and W. Bauhofer, Compos. Sci. Technol., 63, 1835-1841 (2003).

12) T. Takahashi, K. Suzuki, H. Awano and K. Yonetake, Chem. Phys. Lett., 436, 378-382 (2007).

13) H. Wang, H. Zhang, W. Zhao, W. Zhang and G. Chen, Compos. Sci. Technol., 68, 238-243 (2008).

14) T. Takahashi, T. Murayama, A. Higuchi, H. Awano and K. Yonetake, Carbon, 44, 1180-1188 (2006).

15) H.-B. Cho, N. C. Tu, T. Fujihara, S. Endo, T. Suzuki, S. Tanaka, W. Jiang, H. Suematsu, K. Niihara and T. Nakayama, Mater. Lett., 65, 2426-2428 (2011).

16) H.-B. Cho, M. Shoji, T. Fujihara, T. Nakayama, H. Suematsu, T. Suzuki and K. Niihara, J. Ceram. Soc. Japan, 118, 66-69 (2010).

17) H.-B. Cho, Y. Tokoi, S. Tanaka, T. Suzuki, W. Jiang, H. Suematsu, K. Niihara and T. Nakayama, J. Mater. Sci., 46, 2318-2323 (2011).

18) H.-B. Cho, T. Nakayama, Y. Tokoi, S. Endo, S. Tanaka, T. Suzuki, W. Jiang, H. Suematsu and K. Niihara, Compos. Sci. Technol., 70, 1681-1685 (2010).

19) K. B. Shelimov and M. Moskovits, Chem. Mater., 12, 250-254 (2000).

20) M. B. Khusidman and V. S. Neshpor, Poroshkovaya Metallurgiya, 10, 72-77 (1970).

21) H.-B. Cho, Y. Tokoi, S. Tanaka, H. Suematsu, T. Suzuki, W.-H. Jiang, K. Niihara and T. Nakayama, Compos. Sci. Technol., 71, 1046-1052 (2011).

22) P. V. Kamat, K. G. Thomas, S. Barazzouk, G. Girishkumar, K. Vinodgopal and D. Meisel, J. Am. Chem. Soc., 126, $10757-$ 10762 (2004)

23) H.-B. Cho, T. Nakayama, T. Suzuki, S. Tanaka, W. Jiang, H. Suematsu, J.-W. Lee, H.-D. Kim and K. Niihara, Diamond Relat. Mater., 26, 7-14 (2012).

24) T. Thamm, W. Baumann, D. Dietrich, N. Meyer, S. Stoe and G. Marx, Phys. Chem. Chem. Phys., 3, 5150-5153 (2001).

25) H. R. Philipp, Phys. Rev. B, 16, 2896-2900 (1977). 